\title{
miR-433 accelerates acquired chemoresistance of gallbladder cancer cells by targeting cyclin $M$
}

\author{
JIANHUA YU ${ }^{1}$, WEIGUANG ZHANG ${ }^{2}$, BAOCHUN LU $^{1}$, HONGWEI QIAN $^{1}$, \\ HAIJUN TANG ${ }^{1}$, ZHIYANG ZHU ${ }^{1}$, XINGGUI YUAN ${ }^{1}$ and PEITU REN ${ }^{1}$ \\ ${ }^{1}$ Department of Hepatobiliary Surgery, Shaoxing People's Hospital, Shaoxing Hospital of Zhejiang University; ${ }^{2}$ Department of \\ Molecular Medicine and Clinical Laboratory, Shaoxing Second Hospital, Shaoxing, Zhejiang 312000, P.R. China
}

Received January 29, 2017; Accepted October 24, 2017

DOI: $10.3892 / \mathrm{ol} .2017 .7708$

\begin{abstract}
Resistance to chemotherapy is associated with dismal prognosis in patients with gallbladder cancer. Cyclin-dependent kinase 10 (CDK10) influences the chemosensitivity of gallbladder cancer cells, and cyclin $\mathrm{M}$ is the activating factor and binding partner of CDK10. To determine the effect of CDK10 or cyclin M overexpression on chemosensitivity, gemcitabine-resistant (GR) subclones were established from CDK10 or cyclin M stable transfectants. Stable overexpression of CDK10 increased the sensitivity to gemcitabine in non-resistant cells and did not further increase the sensitivity to gemcitabine in the GR subclones. GR subclones exhibited a significantly decreased expression of cyclin $\mathrm{M}$ while maintaining the expression levels of CDK10, compared with the non-resistant cells. MicroRNA (miR)-433 was identified as a candidate factor involved in the mechanism of the downregulation of M cyclin in GR subclones. Luciferase assays confirmed the interaction between miR-433 and the $3^{\prime}$ untranslated region (3'UTR) of cyclin $\mathrm{M}$. Additionally, ectopic expression of miR-433 significantly decreased the expression of cyclin M. Finally, increased expression of circulating miR-433 was associated with poor outcome of chemotherapy. The results of the present study suggest that miR-433 is a potential biomarker for evaluating chemosensitivity in gallbladder cancer.
\end{abstract}

\section{Introduction}

Gallbladder cancer is a relatively rare disease, with an estimated annual incidence of between 2 and 3 individuals/100,000; however, it is the most frequently encountered malignancy of the biliary tract $(1,2)$. Complete surgical resection is the only potentially curative approach in early stages of the disease;

Correspondence to: Dr Weiguang Zhang, Department of Molecular Medicine and Clinical Laboratory, Shaoxing Second Hospital, 123 Yan'an Road, Shaoxing, Zhejiang 312000, P.R. China

E-mail: zwgisme2@126.com

Key words: cyclin-dependent kinase 10, cyclin M, microRNA-433, gallbladder cancer, chemoresistance, c-Raf however, the majority of cases are diagnosed in advanced stages. Additionally, because the aggressive malignancy tolerates traditional chemotherapy and radiotherapy, these cases have a dismal prognosis with an overall 5-year survival rate of $<5 \%$ (3). Therefore, improving the diagnostic accuracy during the early stages and identifying the molecular mechanisms associated with chemotherapy or radiation resistance may provide a potential therapeutic approach for the treatment of gallbladder cancer.

Cyclin M, also known as FAM58A, is a member of the cyclin family, which serves important regulatory functions in the cell cycle and transcription (4). Mutations in cyclin $\mathrm{M}$ cause toe syndactyly, telecanthus and anogenital and renal malformations syndrome, a human developmental anomaly $(4,5)$. However, the function of cyclin $\mathrm{M}$ in other diseases remains unclear. Cyclin $\mathrm{M}$ has been identified as the binding partner for cyclin-dependent kinase 10 (CDK10) (5). CDK10 is involved in the regulation of cell division and function in various types of tumor (6-10). It was confirmed that CDK10 functions as a tumor suppressor gene and regulates the survivability of biliary tract cancer cells, including gallbladder cancer cells (8). These results suggest that cyclin M may also be involved in cancer development through binding to CDK10.

MicroRNAs (miRNAs) are key negative regulators of gene expression in eukaryotes and abundant evidence indicates that miRNAs are involved in human cancers $(11,12)$. miR-433 has been identified as a cancer-associated miRNA in various types of tumor, including bladder cancer, ovarian cancer, gastric cancer, hepatocellular carcinoma and oral squamous cell carcinoma (13-17). Furthermore, aberrant expression levels of miR-433 have been identified to be associated with chemosensitivity and enhanced survival of ovarian cancer cells $(15,18)$.

In the present study, gemcitabine-resistant (GR) subclones were established on the basis of stable transfectants of CDK10 or cyclin M overexpression. To provide a possible mechanism for cyclin $\mathrm{M}$ downregulation in acquired chemoresistance of gallbladder cancer cells, miR-433 was predicted to target cyclin M. Furthermore, the association of serum levels of miR-433 and the chemotherapeutic response in gallbladder cancer was evaluated. Collectively, the results of the present study suggest that aberrant expression of miR-433 affects the chemoresistance of gallbladder cancer cells by targeting cyclin $\mathrm{M}$. 


\section{Materials and methods}

Cell culture, plasmids, RNA oligonucleotides and transfection. The GBC-SD gallbladder carcinoma cell line was obtained from the Cell Bank of Type Culture Collection of the Chinese Academy of Sciences (Shanghai, China). Cells were cultured at $37^{\circ} \mathrm{C}$ in $5 \% \mathrm{CO}_{2}$ in RPMI-1640 medium with $10 \%$ fetal bovine serum (both from Gibco; Thermo Fisher Scientific, Inc., Waltham, MA, USA), $100 \mathrm{IU} / \mathrm{ml}$ penicillin and $100 \mu \mathrm{g} / \mathrm{ml}$ streptomycin. Human CDK10 and cyclin M coding sequences were cloned into pBudCE4.1 vector (Invitrogen; Thermo Fisher Scientific, Inc.). Total RNA was isolated from normal liver tissue using TRIzol reagent (Thermo Fisher Scientific, Inc.) and cDNA was synthesized using the PrimeScript reagent kit (Applied Biosystems; Thermo Fisher Scientific, Inc.), according to the manufacturer's instructions. The primer sequences for PCR amplification of CDK10-open reading frame (ORF) were as follows: 5'-CCAAGCTTATGGCGG AGCCAGATCTGGA-3' (forward) and 5'-CCGGATCCT CAGGGTTTACAGCGCTTG-3' (reverse). The PCR products were purified and cloned into the HindIII/BamHI sites of the vector. The primer sequences for PCR amplification of cyclin M-ORF were as follows: 5'-GGGTACCATGGA AGCCCCGGAGGG-3' (forward) and 5'-CCCTCGAGT TAGGGGATCTCTGTGTCCA-3' (reverse). The PCR products were purified and cloned into the $K p n \mathrm{I} / \mathrm{XhoI}$ sites of the vector. CDK10-ORF was cloned into the pBudCE4.1 vector and cyclin M-ORF was cloned into the pBudCE4.1 vector to produce pBudCE4.1-CDK10 and pBudCE4.1-CycM, respectively. Finally, CDK10-ORF was cloned into the pBudCE4.1-CycM vector and named pBudCE4.1-Co. All the constructs were confirmed by DNA sequencing and the pBudCE4.1 empty vector was used as a vector control.

GBC-SD cells were transfected with pBudCE4.1-CDK10, pBudCE4.1-CycM, pBudCE4.1-Co or empty vector plasmid using Lipofectamine ${ }^{\circledR} 2000$ (Invitrogen; Thermo Fisher Scientific, Inc.). At $48 \mathrm{~h}$ post-transfection, the culture medium was replaced with medium containing Zeocin (Invitrogen; Thermo Fisher Scientific, Inc.) and the medium containing Zeocin was replaced every 3-4 days. After 2 weeks, isolated colonies began to appear. After 3 weeks, stable transfectants expressing CDK10 or cyclin $\mathrm{M}$ were selected for further study. The control clones expressing the empty vector were isolated at the same time. The stable transfectants were named as follows: GBC-Mock, GBC-CDK10, GBC-CycM and GBC-Co, respectively.

Small interfering RNA (siRNA) targeting the proto-oncogene serine/threonine-protein kinase c-Raf was obtained from RiboBio (Guangzhou, China) and the sequence was 5'-GCUGCAUCUCUCCUACAAU-3'. Control siRnA was obtained from RiboBio (8). Other RNA oligonucleotides (including miR-433, anti-miR-433, miR-control and siRNA-control groups) were also obtained from RiboBio. At $24 \mathrm{~h}$ before transfection, cells were plated onto a $35-\mathrm{mm}$ dish at 50-60\% confluence. RNA oligonucleotides transfection was performed at $50 \mathrm{nM}$ using Lipofectamine ${ }^{\circledR} 2000$, according to the manufacturer's instructions. The transfected cells were resuspended in RPMI-1640 medium and cultured for 48-72 h before analysis. Each assay was performed in triplicate at least.
Establishment of GR subclones. Stable transfectants were exposed to increasing concentrations $(0.001,0.0025,0.005$, $0.01,0.025,0.05,0.1,0.25,0.3,0.35,0.4,0.45$ and $0.5 \mu \mathrm{g} / \mathrm{ml})$ of gemcitabine (Lilly France S.A., Lilly, Fegersheim, France) every 4 weeks over a 9-month period. As cells adapted to the doses, the gemcitabine concentration was increased. The cell subclones were named as follows: GBC-Mock-GR, GBC-CDK10-GR, GBC-CycM-GR and GBC-Co-GR.

Western blot analysis. Total proteins were extracted from tissue samples or cells using lysis buffer containing phenylmethyl sulfonylfluoride (both from Beyotime Institute of Biotechnology, Haimen, China) at $25^{\circ} \mathrm{C}$ and protein concentration was determined by BCA Protein Assay kit (Beyotime Institute of Biotechnology). A total of $20 \mu \mathrm{g}$ protein was separated by SDS-PAGE (10\% gel) and transferred onto a polyvinylidene fluoride membrane. Membranes were blocked with 5\% non-fat milk and incubated with anti-CDK10 (cat. no. 17182-1-AP, 1:500; ProteinTech Group, Inc., Chicago, IL, USA), anti-c-Raf (cat. no. AP7816d, 1:500; Abgent, Inc., San Diego, CA, USA,) and anti-cyclin M (cat. no. ab81062, 1:1,000; Abcam, Cambridge, UK) primary antibodies at $4^{\circ} \mathrm{C}$ for 12 h. Anti- $\beta$-actin (cat. no. sc-47778, 1:1,000; Santa Cruz Biotechnology, Inc., Dallas, TX, USA) was used as a loading control. Membranes were then washed and incubated with horseradish peroxidase-conjugated secondary antibody (cat. no. A21020 and A21010, 1:8,000; Abbkine, Wuhan, China). Immunoreactive bands were visualized using a chemiluminescence solution with an ECL kit (Beyotime Institute of Biotechnology). Quantity One (Bio-Rad Laboratories, Inc., Hercules, CA, USA) was used as the software for quantification.

Cell counting Kit-8 (CCK-8) assay. Cells were seeded into a 96-well plate at a density of $4 \times 10^{3}$ cells/well, and allowed to attach at $37^{\circ} \mathrm{C}$ overnight. Cells were treated with various concentrations $(0,0.01,0.05,0.1,0.5,1$ and $10 \mu \mathrm{g} / \mathrm{ml})$ of gemcitabine in $\geq 6$ replicate wells and incubated at $37^{\circ} \mathrm{C}$ for $48 \mathrm{~h}$. Cell proliferation was determined using the CCK- 8 assay (Beyotime Institute of Biotechnology). In brief, a 1/10 volume of the CCK- 8 solution was added to each well, and the cells were cultured for another $2 \mathrm{~h}$. Cell viability was determined by measuring the absorbance at $450 \mathrm{~nm}$ using a plate reader (BioTek Instruments, Inc., Winooski, VT, USA).

Colony formation assay. Cells were seeded for colony formation in 24-well dishes at a density of 500 viable cells/well. The medium contained $1 \mu \mathrm{g} / \mathrm{ml}$ gemcitabine and was exchanged every 2 days. After 14 days, the cells were fixed in $4 \%$ paraformaldehyde for $15 \mathrm{~min}$. Following washing, the cells were stained with $0.005 \%$ crystal violet solution for $1 \mathrm{~h}$. The plates were aspirated, washed and allowed to air dry. The dishes were photographed with a camera and the colonies were counted (a single colony contained $>20$ cells) using a light microscope (magnification, $\mathrm{x} 40$. Each assay was performed in triplicate.

Clinical tissue and blood samples. Gallbladder cancer tissue and paired normal tissue specimens were obtained from 8 patients at the Shaoxing People's Hospital (Shaoxing, China) from September 2012 to March 2016. All participants 
Table I. Primers designed for cyclin M, CDK10 and $\beta$-actin.

\begin{tabular}{llll}
\hline Name & Symbol & \multicolumn{1}{c}{ Forward (5'-3') } & \multicolumn{1}{c}{ Reverse (5'-3') } \\
\hline$\beta$-actin & ACTB & AGAAGGAGATCACTGCCCTGGCACC & CCTGCTTGCTGATCCACATCTGCTG \\
CDK10-ORF & CDK10 & TGGACAAGGAGAAGGATG & CTGCTCACAGTAACCCATC \\
CDK10-3'UTR & CDK10 & GTGCAACCACTGCTTCTTGG & AGCGTCCACCACAACTCAAA \\
Cyclin M-ORF & FAM58A & ACTTCCGAGTGGCGAGGTTCAT & TCATAGGCGTCCAGGTTGGTCT \\
Cyclin M-3'UTR & FAM58A & CGCTTTGCCAGTCCCTAGAA & GACAAACGCAGATGTGGCTG \\
\hline
\end{tabular}

CDK10, cyclin-dependent kinase 10; ORF, open reading frame; 3'UTR, 3' untranslated region.

provided written informed consent and the tissue acquisition protocol was approved by the Zhejiang University Institutional Review Board (Hangzhou, China). Blood samples were collected from 23 patients with gallbladder cancer and the survival time was $>3$ months. Among the patients, 17 patients underwent chemotherapy with gemcitabine or 5-fluorouracil and were assigned to the chemotherapy group. The remaining 6 patients did not receive chemotherapy and were assigned to the non-chemotherapy group. Blood samples from the patients were collected and serum was separated by centrifugation $\left(1,000 \mathrm{x} \mathrm{g}\right.$, for $5 \mathrm{~min}$ at $\left.4^{\circ} \mathrm{C}\right)$ collected into RNase/DNase-free tubes and stored at $-80^{\circ} \mathrm{C}$ until use. Fresh tissues were stored at $-80^{\circ} \mathrm{C}$.

Reverse transcription-quantitative polymerase chain reaction (RT-qPCR). Total RNA was isolated from cells, tissues or serum using TRIzol reagent, and RNA was reverse-transcribed into cDNA using a High Capacity cDNA Reverse Transcription kit (Takara Biotechnology Co., Ltd., Dalian, China), according to the manufacturer's instructions. qPCR was performed using SYBR Green PCR Master Mix (Takara Biotechnology Co., Ltd.) and the ABI 7500 Real-time PCR system (Applied Biosystems; Thermo Fisher Scientific, Inc.), according to the manufacturer's instructions. Specific primers were designed for cyclin M, CDK10 and $\beta$-actin (Table I). The PCR conditions included $95^{\circ} \mathrm{C}$ for $5 \mathrm{~min}$, and a total of 40 cycles of 95,60 and $72^{\circ} \mathrm{C}$ for $30 \mathrm{sec}$ and then a final extension at $72^{\circ} \mathrm{C}$ for $5 \mathrm{~min}$. $\beta$-actin was used as an endogenous control. miR-433 normalization was performed according to a protocol described previously (19). Expression of miR-433 was normalized using U6 RNA (for RNA from cells and tissues) and with Caenorhabditis elegans miR-39 mimic (for RNA from culture supernatant and serum samples) which was used as an RNA spike-in. Specific primers for miR-433, U6 and C. elegans miR-39 RNA were obtained from RiboBio $(15,19)$. In total, three individual experiments were performed. Relative expression levels of miR-433 were determined using the $2^{-\Delta \Delta \mathrm{Cq}}$ method (20).

Dual-luciferase reporter assay. Predictions for the potential binding sites of miR-433 in the 3'UTR of cyclin M was performed using 2 different online programs: TargetScan 6.2 (http://www.targetscan.org/vert_61/) and microRNA.org (http://www.microrna.org). The 3'UTR of cyclin $\mathrm{M}$ was amplified by PCR using human genomic DNA as a template. The primers used were as follows: 5'-CGACGC
GTTTGACTACAATACAGGCATGACA-3' (forward) and 5'-CCAAGCTTTCCGTAGGGATTCAGTCTCA-3' (reverse). The PCR product, which contained a potential binding site for miR-433, was digested with MluI and HindIII, and was inserted downstream of the luciferase gene in the pMIR-REPORT system (Applied Biosystems; Thermo Fisher Scientific, Inc.). The constructs were verified by DNA sequencing. The verified construct was named pMIR-CycM-3'UTR. The mutant reporter plasmids were constructed in accordance with the aforementioned criteria by annealing the following oligonucleotides: 5'-CGACGCGTTTGACTACAATACAGGCAT GACATCAATGAAAGGAAAGTC catAAATCGATGAGA CTGAATCCCTACGGAAAGCTTGG-3' and 5'-CCAAGC TTTCCGTAGGGATTCAGTCTCATCGATTT atgGAC TTTCCTTTCATTGATGTCATGCCTGTATTGTAGTCA

AACGCGTCG-3' (the bases in lower-case indicate the site mutations). The mutant reporter plasmids were named mut-pMIR-CycM-3'UTR. For luciferase assays, 293T cells were cultured in 6-well plates and transfected with plasmids (including pMIR-CycM-3'UTR, mut-pMIR-CycM-3'UTR and $\beta$-galactosidase reporter plasmids) and RNA oligonucleotides using Lipofectamine ${ }^{\circledR}$ 2000. A $\beta$-galactosidase reporter plasmid was designed to normalize transfection efficiency. At $48 \mathrm{~h}$ after transfection, the cells were lysed, luciferase activity was determined using a Dual-Luciferase Reporter assay system and $\beta$-galactosidase activity was determined using the $\beta$-Galactosidase Enzyme assay system (both from Promega Corporation, Madison, WI, USA).

Statistical analysis. Data were analyzed using SPSS software (version 13.0; SPSS, Inc., Chicago, IL, USA). The relevant data are expressed as the mean \pm standard deviation (SD). Statistical significance between two groups was determined using Student's t-test. One-way analysis of variance followed by a Tukey-Kramer test was used to examine differences among multiple groups. $\mathrm{P}<0.05$ was considered to indicate a statistically significant difference.

\section{Results}

Decreasing expression of cyclin $M$ contributes to acquired chemoresistance of GR subclones. To generate stable cell lines for experimental analyses, GBC-SD cells were transfected with pBudCE4.1-CDK10, pBudCE4.1-CycM, pBudCE4.1-Co or with the empty vector plasmid. After 4 weeks, stable transfectants were obtained and named GBC-CDK10, GBC-CycM, 

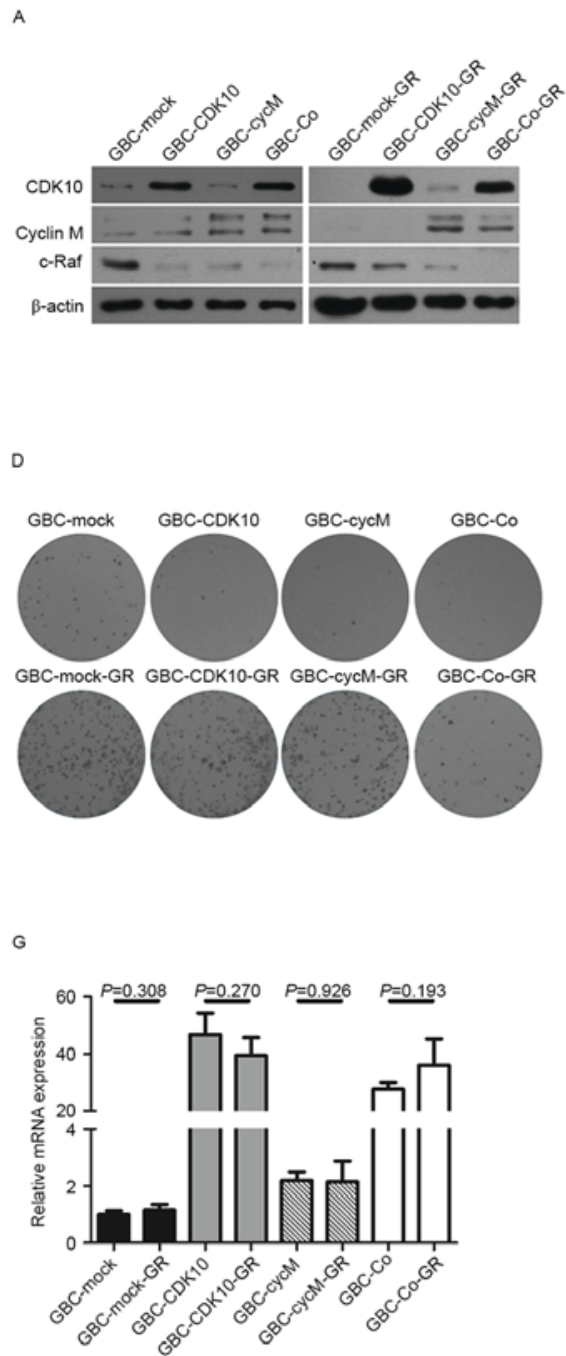

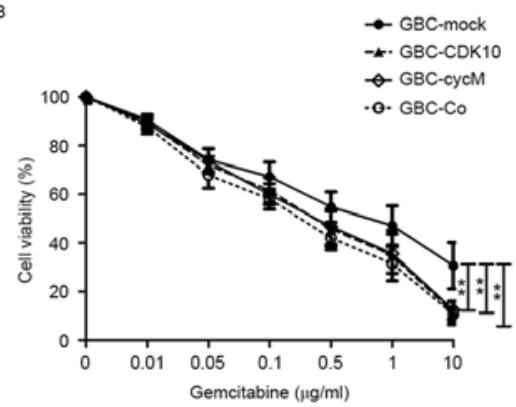

E
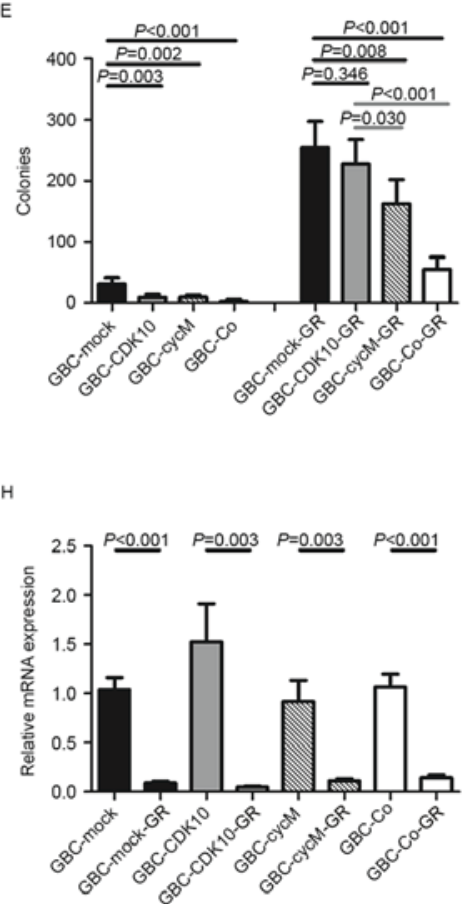

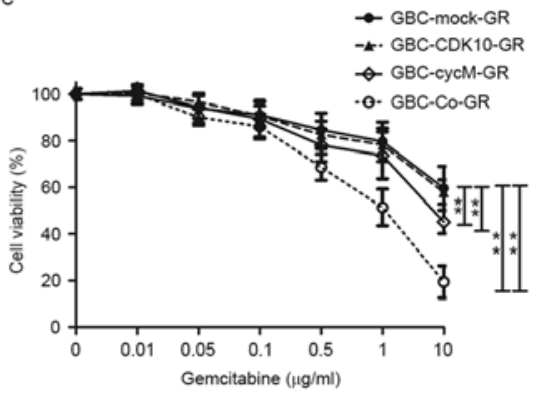

.
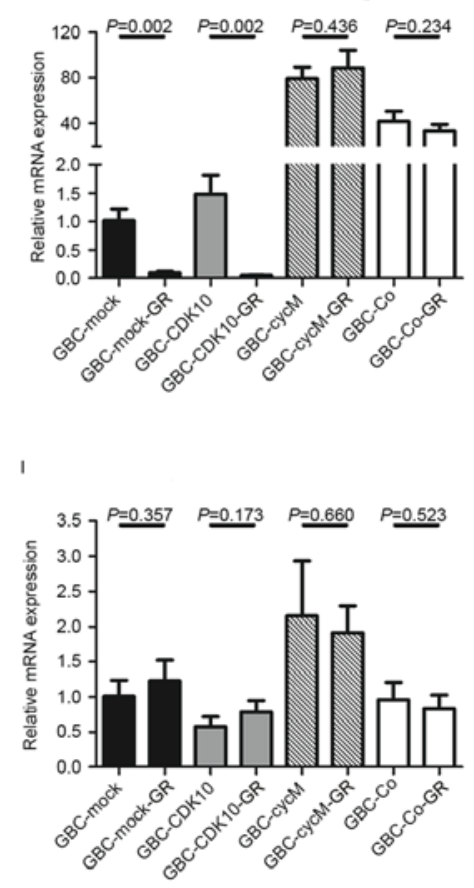

Figure 1. Aberrant expression of cyclin M is associated with the chemoresistance of gallbladder cancer cells. (A) Western blot analysis of CDK10, cyclin M and c-Raf expression in each cell line. $\beta$-actin was used as a loading control. (B and C) Dose-response curves exhibiting the cell viability (expressed as a percentage) of (B) non-GR subclones or (C) GR subclones following exposure to gemcitabine. (D) Colony forming assay. Representative images of the various cell lines in response to $1 \mu \mathrm{g} / \mathrm{ml}$ gemcitabine. (E) Number of colonies in each cell line in response to $1 \mu \mathrm{g} / \mathrm{ml}$ gemcitabine. (F) Relative mRNA expression of the ORF of cyclin M in each cell line using PCR. (G) Relative mRNA expression of the ORF of CDK10 in each cell line using PCR. (H) Relative mRNA expression of the $3^{\prime} \mathrm{UTR}$ of cyclin M in each cell line using PCR. (I) Relative mRNA expression of the $3^{\prime} \mathrm{UTR}$ of CDK10 in each cell line using PCR. *"P<0.01. GR, gemcitabine-resistant; CDK10, cyclin-dependent kinase 10; ORF, open reading frame; 3'UTR, 3' untranslated region; GBC, gallbladder cancer; PCR, polymerase chain reaction; CycM, cyclin M; Co, transfected with the plasmids which contained CDK10-ORF and CycM-ORF.

GBC-Co and GBC-Mock, respectively. Western blot analysis was performed to confirm positive clones (Fig. 1A). Next, the chemosensitivity of the four stable cell lines to gemcitabine was examined. The results demonstrated that GBC-CDK10 and GBC-CycM cells exhibited a significantly increased sensitivity to gemcitabine (Fig. 1B). As expected, GBC-Co cells were also more sensitive to gemcitabine than were GBC-Mock cells (Fig. 1B).

Next, the four stable cell lines (GBC-CDK10, GBC-CycM, GBC-Co and GBC-Mock) were cultured for 9 months in the presence of gemcitabine to establish GR subclones (Fig. 1B and C). The dose-response curves indicated that GBC-CDK10-GR cells exhibited a similar chemosensitivity to that of the GBC-Mock-GR cells and were more resistant to gemcitabine compared with the GBC-Co-GR or GBC-CycM-GR cells (Fig. 1C). Following extended exposure to gemcitabine, although GBC-CDK10-GR cells maintained high levels of CDK10 expression (Fig. 1A),
GBC-CDK10-GR cells did not exhibit the increased sensitivity to gemcitabine that was exhibited by GBC-CDK10 cells (Fig. 1C).

Additionally, colony formation assays were performed in all four-cell lines, which were cultured with $1 \mu \mathrm{g} / \mathrm{ml}$ gemcitabine. After 14 days, GBC-CDK10, GBC-CycM and GBC-Co cells were more sensitive to gemcitabine compared with GBC-Mock cells, and GBC-Co-GR cells were more sensitive to gemcitabine compared with GBC-Mock-GR and GBC-CDK10-GR cells (Fig. 1D and E). These results confirm the results obtained from the dose-response curves (Fig. 1B and $\mathrm{C}$ ).

Cyclin $\mathrm{M}$ is a binding partner for CDK10 (5). It was demonstrated that cyclin $\mathrm{M}$ protein levels were too low to detect by western blot in the GBC-CDK10-GR cells (Fig. 1A), different from the GBC-CDK10 cells. It suggested that decreasing expression of cyclin $\mathrm{M}$ may contribute to the decreased chemosensitivity of CDK10-overexpressing GBC-SD cells. 


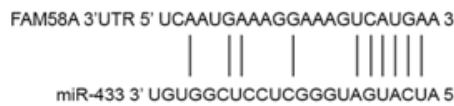

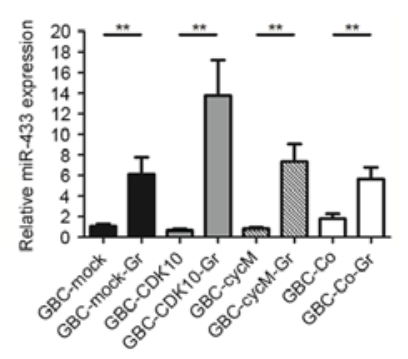

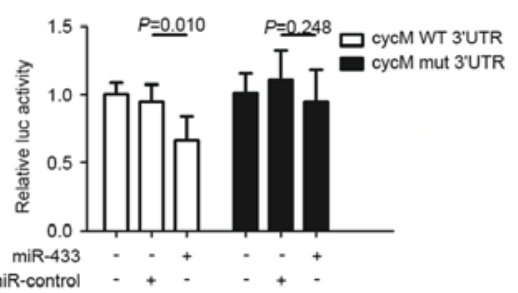

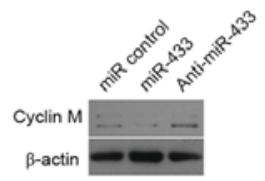

E

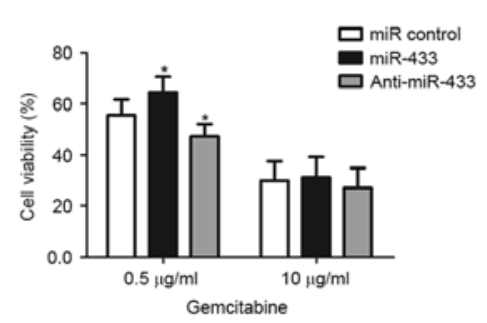

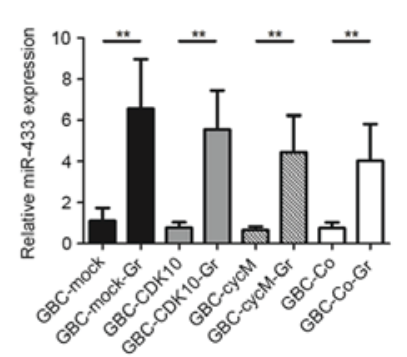

$G$

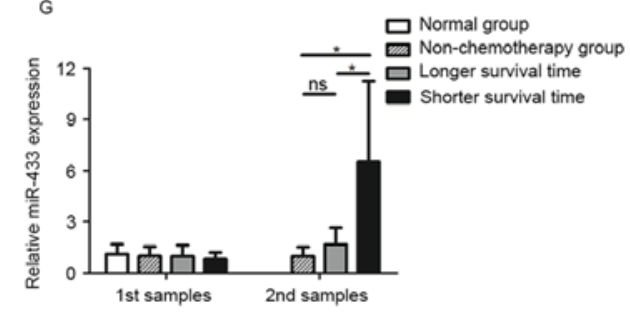

Figure 2. miR-433 accelerates acquired chemoresistance of gallbladder cancer cells by targeting cyclin M. (A) Putative miR-433-binding sequences in the 3'UTR of cyclin M (FAM58A) mRNA. (B) Relative expression of miR-433 in GR and non-GR subclones. (C) Relative luciferase activity in CycM WT 3'UTR (cells were transfected with pMIR-CycM-3'UTR) and CycM mut 3'UTR (cells were transfected with mut-pMIR-CycM-3'UTR)-293T cells. $\beta$-galactosidase activity was used as a normalization control. (D) Western blot analysis of cyclin M expression in GBC-SD cells following transfection with miR-433 or anti-miR-433. $\beta$-actin was used as a loading control. (E) Cell viability (expressed as a percentage) of GBC-SD cells following transfection with miR-433 or anti-miR-433 in response to 0.5 and $1 \mu \mathrm{g} / \mathrm{ml}$ gemcitabine. (F) Relative expression of miR-433 in supernatants from GR and non-GR subclones. (G) Relative expression of miR-433 in serum samples. Patients in the longer survival time group and shorter survival time group underwent chemotherapy with gemcitabine or 5-fluorouracil. The first samples were collected from the patients prior to receiving chemotherapy and the second samples were collected following three chemotherapy cycles. ${ }^{*} \mathrm{P}<0.05 ;{ }^{* *} \mathrm{P}<0.01$. GR, gemcitabine-resistant; CDK10, cyclin-dependent kinase 10; 3'UTR, 3' untranslated region; miR, microRNA; luc, luciferase; GBC, gallbladder cancer; CycM, cyclin M; WT, wild-type; mut, mutant; Co, transfected with the plasmids which contained CDK10-ORF and CycM-ORF.

Because of the transfection, both the transcripts from plasmid and genome could influence the protein levels of CDK10 and cyclin M. The exogenous transcription product (from plasmid) could cover up the practical change of endogenous expression. Considering that only the open reading frames (ORFs), without 3'UTRs, were cloned into plasmid in our study, the expression of the 3'UTRs may more accurately reflect the endogenous expression of CDK10 and cyclin M (Fig. 1F-G). To perform PCR, specific primers for the 3'UTRs of CDK10 and cyclin M were designed. The results demonstrated that the 3'UTR of cyclin M was significantly decreased in GR subclones (Fig. 1H). However, the 3'UTR of CDK10 did not exhibit a similar change (Fig. 1I). This result indicates that downregulating the expression of cyclin M may be an important mechanism for the acquired resistance in GR subclones.

Cyclin $M$ is a target of miR-433 in gallbladder cancer cells. The interactions between miRNAs and the 3'UTRs of target genes are key regulatory mechanisms that are involved in multiple biological processes and human diseases, including cancer (21-23). To examine the molecular mechanism underlying cyclin $\mathrm{M}$ downregulation in gallbladder cancer cells, miRNAs targeting cyclin M using the TargetScan 6.2 and microRNA.org software were employed. Considering the association between candidate miRNAs and chemoresistance $(15,18)$, miR-433 was hypothesized to act as a candidate target for cyclin M. miR-433-binding sequences in the 3'UTR of cyclin M mRNA are indicated in Fig. 2A. Examination of miR-433 expression levels in the GBC subclones revealed that all four GR subclones, i.e., GBC-Mock-GR, GBC-CDK10-GR, GBC-CycM-GR and GBC-Co-GR, expressed significantly increased levels of miR-433 compared with their respective non-GR subclones (Fig. 2B).

Luciferase activity assays indicated that the miR-433 mimic repressed the luciferase activity of pMIR-CycM-3'UTR reporter plasmid, but did not repress the activity of mut-pMIR-CycM-3'UTR (Fig. 2C). The miR-control had no impact on the luciferase activity of pMIR-CycM-3'UTR (Fig. 2C). To further confirm the results, cyclin M expression levels were investigated following transfection with miR-433 in GBC-SD cells. Western blot analysis was performed at $72 \mathrm{~h}$ post-transfection. It was revealed that cyclin $\mathrm{M}$ was downregulated in GBC-SD cells following miR-433 transfection, whereas cyclin $\mathrm{M}$ was upregulated following transfection with anti-miR-433 (Fig. 2D).

miR-433 expression enhances the chemoresistance of gallbladder cancer cells. Considering that miR-433 was expressed at a high level in GR subclones and targets cyclin M, the function of miR-433 in promoting resistance to gemcitabine in GBC-SD cells was examined in vitro. The results indicated that the miR-433 mimic enhanced the resistance of GBC-SD cells in response to a moderate dose of gemcitabine $(0.5 \mu \mathrm{g} / \mathrm{ml})$, whereas anti-miR-433 treatment reversed this change (Fig. 2E). However, in response to a high gemcitabine dose $(10 \mu \mathrm{g} / \mathrm{ml})$, 


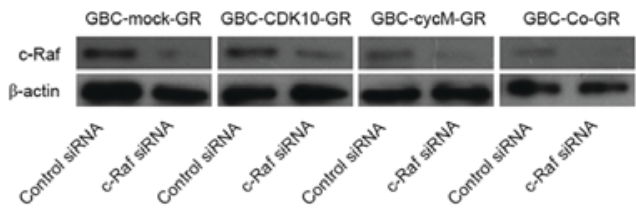

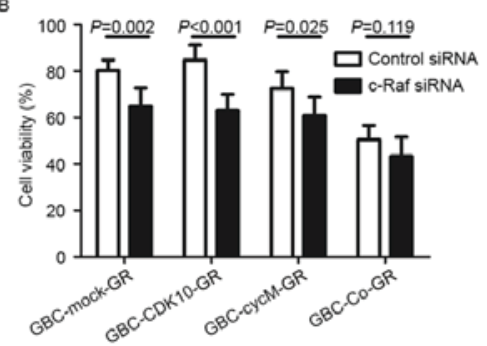

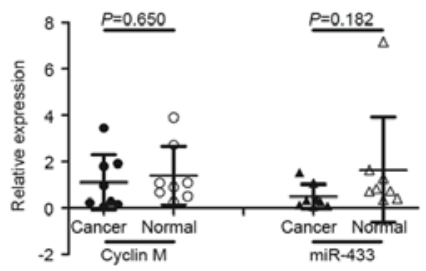

Figure 3. Increased expression of c-Raf induced by downregulated cyclin M expression is a potential mechanism to increased chemoresistance. (A) Western blot analysis of c-Raf expression in c-Raf-silenced or non-c-Raf-silenced cells. $\beta$-actin was used as a loading control. (B) Cell viability (expressed as a percentage) of c-Raf-silenced or non-c-Raf-silenced subclones in response to $1 \mu \mathrm{g} / \mathrm{ml}$ gemcitabine. (C) Relative expression level of cyclin $\mathrm{M}$ and miR-433 in tissue samples from patients with gallbladder cancer $(n=8)$. GR, gemcitabine-resistant; CDK10, cyclin-dependent kinase 10; miR, microRNA; siRNA, small interfering RNA; GBC, gallbladder cancer; CycM, cyclin M; Co, transfected with the plasmids which contained CDK10-ORF and CycM-ORF.

the function of the miR-433 mimic or inhibitor did not induce any significant effect.

Circulating miR-433 levels are associated with chemotherapy in gallbladder cancer. A previous study demonstrated that ovarian cancer cells with increased miR-433 expression are able to release miR-433 into the growth medium (15). Therefore, the relative miR-433 expression was examined in the culture supernatant of the subclones. The results indicated that the culture supernatant from GR subclones exhibited significantly increased relative miR-433 expression compared with that from non-GR subclones (Fig. 2F).

Serum expression levels of miR-433 were examined in patients with gallbladder cancer, from the chemotherapy group and the non-chemotherapy group. The chemotherapy group comprised 17 patients with a median survival time of 9.8 months. Among these patients, 9 patients had a shorter survival time compared with the median (shorter survival time group, as presented in Fig. 2G) and the remaining 8 patients had a longer survival time compared with the median (longer survival time group, as presented in Fig. 2G). The first serum samples were collected from the patients prior to receiving chemotherapy. The results indicated no marked difference in miR-433 levels between the non-chemotherapy group and chemotherapy group, regardless of shorter or longer survival time (Fig. 2G). Following three chemotherapy cycles, additional serum samples were collected. Interestingly, the patients in the chemotherapy group with a shorter survival time exhibited significantly increased levels of miR-433 in the serum, compared with the patients in the non-chemotherapy group or with the patients in the chemotherapy group with a longer survival time (Fig. 2G). However, although they underwent chemotherapy, the patients with a longer survival time did not exhibit higher miR-433 levels in the serum compared with those in the non-chemotherapy group.

Increased expression of c-Raf induced by dysfunction of CDK10-cyclin M increases chemoresistance in gallbladder cancer cells. Considering that the CDK10-cyclin M complex regulates the expression of c-Raf $(5,6)$, c-Raf protein levels were examined prior to and following the establishment of GR subclones. The results demonstrated that c-Raf protein expression was markedly increased in cells with decreased expression of CDK10 or decreased expression of cyclin M
(Fig. 1E). However, the expression of c-Raf was at low levels under conditions of increased expression of CDK10 and cyclin $\mathrm{M}$, such as in the case of GBC-Co cells and GBC-Co-GR cells (Fig. 1E).

Subsequently, the involvement of c-Raf to chemoresistance was assessed. All GR subclones, including GBC-Mock-GR, GBC-CDK10-GR, GBC-CycM-GR and GBC-Co-GR, were transfected with siRNA against c-Raf and c-Raf downregulation was confirmed by western blot analysis (Fig. 3A). The results demonstrated that c-Raf silencing significantly increased the sensitivity to gemcitabine in GBC-Mock-GR, GBC-CDK10-GR and GBC-CycM-GR cells (Fig. 3B). Therefore, increased expression of $\mathrm{c}$-Raf is also an important factor that is involved in the regulation of chemoresistance in cancer.

\section{Discussion}

Resistance to chemotherapy is a major concern in gallbladder cancer (3). Acquired chemoresistance of cancer cells may arise following extended exposure to chemotherapy drugs. The majority of patients with gallbladder cancer that undergo chemotherapy become resistant following consecutive treatments and fail to benefit from chemotherapy (24). Therefore, it is important to clarify the molecular mechanism underlying acquired chemoresistance for an improved treatment of gallbladder cancer.

Gemcitabine is a deoxycytidine analogue with antitumor activity and a key drug for chemotherapy of biliary tract cancer $(25,26)$. To investigate the acquired chemoresistance of gallbladder cancer cells, GR subclones were established. Drug concentrations were gradually increased to establish subclones of GBC-SD cells that were able to grow in medium with $0.5 \mu \mathrm{g} / \mathrm{ml}$ gemcitabine. This strategy may simulate the process of acquired chemoresistance. Although CCK-8 or MTT assays are used to examine sensitivity to chemotherapy during a relatively short period ( 48 or $72 \mathrm{~h}$ ), a colony formation assay is able to accurately determine chemosensitivity and colony forming ability under extended exposure to gemcitabine ( 2 weeks).

Similar to a previous study (8), the results of the present study confirmed that GBC-CDK10 cells were more sensitive to gemcitabine compared with GBC-Mock cells. Although GBC-CDK10-GR and GBC-CDK10 cells expressed high levels of CDK10, the gemcitabine resistance of GBC-CDK10-GR cells was as high as that of GBC-Mock-GR cells in contrast 
with that of GBC-CDK10 cells. This indicates that the expression level of CDK10 alone was not able to explain the acquired gemcitabine resistance of GBC-SD cells. However, GBC-Co-GR cells and GBC-Co cells, which expressed increased CDK10 and cyclin M, exhibited a relatively increased sensitivity to gemcitabine compared with that of GBC-Mock-GR and GBC-Mock cells. Additionally, the levels of cyclin M mRNA that contained the 3'UTR were significantly downregulated in GR subclones, compared with that observed in the non-GR subclones This suggests that cyclin M serves an important function in the acquired gemcitabine resistance of gallbladder cancer cells.

miRNAs regulate the expression of target genes by interacting with the 3'UTRs of the mRNA. The results of the present study demonstrated that the 3'UTR of cyclin M was downregulated in all GR subclones and miR-433 was identified as a predicted miRNA to target cyclin M. Overexpression of miR-433 was demonstrated to promote resistance to paclitaxel in ovarian cancer cells (15). The results of the present study also demonstrated that the acquired gemcitabine resistance of gallbladder cancer cells was associated with an upregulation of miR-433. However, other studies reported that miR-433 functions as an anti-oncomiR in various tumors by regulating various targets $(13,14,27,28)$. The results of the present study demonstrated that the expression of miR-433 was decreased in the tumor samples, but this decrease was not statistically significant. Furthermore, the expression of cyclin M between tumor samples and normal tissues also did not exhibit a significant difference. The expression of miR-433 in serum samples was also examined. No differences in the expression of miR-433 were identified in the serum of normal volunteers and patients with gallbladder cancer. Nevertheless, the patients with poor response to chemotherapy exhibited a significant increase in the expression of serum miR-433, whereas the patients with an improved response did not exhibit changes in the serum levels of miR-433. These observations indicate that circulating miR-433 expression may not be useful as a biomarker for diagnosis, but it may be a potential biomarker to evaluate the response to chemotherapy.

The CDK10-cyclin M complex is a negative regulator of c-Raf $(5,6)$. The results of the present study also identified that downregulated cyclin M expression or downregulated CDK10 expression resulted in increasing c-Raf expression. Considering that $\mathrm{c}-\mathrm{Raf}$ silencing reversed the resistance to gemcitabine in GBC-Mock-GR, GBC-CDK10-GR and GBC-CycM-GR cells, the increasing gemcitabine resistance of GBC-SD cells may be attributed to upregulated c-Raf expression, which was induced by downregulation of cyclin $\mathrm{M}$ expression.

In conclusion, the results of the present study suggest that the miR-433/cyclin $\mathrm{M}$ axis is associated with the acquired chemoresistance of gallbladder cancer cells. Circulating miR-433 expression represents a potential biomarker for evaluating the chemosensitivity of gallbladder cancer cells.

\section{Acknowledgements}

The present study was supported by Zhejiang Provincial Natural Science Foundation of China (grant no. LQ14H160001), National Natural Science Foundation of China (grant no. 81602044), Zhejiang Provincial Public Welfare Technology
Application Research Projects (grant nos. 2013C33214 and 2015C33293) and Public Welfare Technology Application Research Projects of Shaoxing (grant no. 2014B70073).

\section{References}

1. Ferlay J, Shin HR, Bray F, Forman D, Mathers C and Parkin DM: Estimates of worldwide burden of cancer in 2008: GLOBOCAN 2008. Int J Cancer 127: 2893-2917, 2010.

2. Lai CH and Lau WY: Gallbladder cancer - a comprehensive review. Surgeon 6: 101-110, 2008.

3. Goetze TO: Gallbladder carcinoma: Prognostic factors and therapeutic options. World J Gastroenterol 21: 12211-12217, 2015.

4. Unger S, Böhm D, Kaiser FJ, Kaulfuss S, Borozdin W, Buiting K, Burfeind P, Böhm J, Barrionuevo F, Craig A, et al: Mutations in the cyclin family member FAM58A cause an X-linked dominant disorder characterized by syndactyly, telecanthus and anogenital and renal malformations. Nat Genet 40: 287-289, 2008.

5. Guen VJ, Gamble C, Flajolet M, Unger S, Thollet A, Ferandin Y, Superti-Furga A, Cohen PA, Meijer L and Colas P: CDK10/cyclin M is a protein kinase that controls ETS2 degradation and is deficient in STAR syndrome. Proc Natl Acad Sci USA 110: 19525-19530, 2013.

6. Iorns E, Turner NC, Elliott R, Syed N, Garrone O, Gasco M, Tutt AN, Crook T, Lord CJ and Ashworth A: Identification of CDK10 as an important determinant of resistance to endocrine therapy for breast cancer. Cancer Cell 13: 91-104, 2008.

7. Leman ES, Magheli A, Yong KM, Netto G, Hinz S and Getzenberg RH: Identification of nuclear structural protein alterations associated with seminomas. J Cell Biochem 108: 1274-1279, 2009

8. Yu JH, Zhong XY, Zhang WG, Wang ZD, Dong Q, Tai S, Li H and Cui YF: CDK10 functions as a tumor suppressor gene and regulates survivability of biliary tract cancer cells. Oncol Rep 27: 1266-1276, 2012.

9. Zhong XY, Xu XX, Yu JH, Jiang GX, Yu Y, Tai S, Wang ZD and Cui YF: Clinical and biological significance of Cdk10 in hepatocellular carcinoma. Gene 498: 68-74, 2012.

10. Kasten M and Giordano A: Cdk10, a Cdc2-related kinase, associates with the Ets2 transcription factor and modulates its transactivation activity. Oncogene 20: 1832-1838, 2001.

11. Lee RC and Ambros V: An extensive class of small RNAs in Caenorhabditis elegans. Science 294: 862-864, 2001.

12. Yates LA, Norbury CJ and Gilbert RJ: The long and short of microRNA. Cell 153: 516-519, 2013.

13. Xu X, Zhu Y, Liang Z, Li S, Xu X, Wang X, Wu J, Hu Z, Meng S, Liu B, et al: c-Met and CREB1 are involved in miR-433-mediated inhibition of the epithelial-mesenchymal transition in bladder cancer by regulating Akt/GSK-3 $\beta /$ Snail signaling. Cell Death Dis 7: e2088, 2016.

14. Wang XC, Ma Y, Meng PS, Han JL, Yu HY and Bi LJ: miR-433 inhibits oral squamous cell carcinoma (OSCC) cell growth and metastasis by targeting HDAC6. Oral Oncol 51: 674-682, 2015.

15. Weiner-Gorzel K, Dempsey E, Milewska M, McGoldrick A, Toh V, Walsh A, Lindsay S, Gubbins L, Cannon A, Sharpe D, et al: Overexpression of the microRNA miR-433 promotes resistance to paclitaxel through the induction of cellular senescence in ovarian cancer cells. Cancer Med 4: 745-758, 2015.

16. Yang Z, Tsuchiya H, Zhang Y, Hartnett ME and Wang L: MicroRNA-433 inhibits liver cancer cell migration by repressing the protein expression and function of cAMP response element-binding protein. J Biol Chem 288: 28893-28899, 2013.

17. Guo LH, Li H, Wang F, Yu J and He JS: The tumor suppressor roles of miR-433 and miR-127 in gastric cancer. Int J Mol Sci 14: 14171-14184, 2013

18. Furlong F, Fitzpatrick P, O'Toole S, Phelan S, McGrogan B, Maguire A, O'Grady A, Gallagher M, Prencipe M, McGoldrick A, et al: Low MAD2 expression levels associate with reduced progression-free survival in patients with high-grade serous epithelial ovarian cancer. J Pathol 226: 746-755, 2012.

19. Kroh EM, Parkin RK, Mitchell PS and Tewari M: Analysis of circulating microRNA biomarkers in plasma and serum using quantitative reverse transcription-PCR (qRT-PCR). Methods 50: 298-301, 2010.

20. Livak KJ and Schmittgen TD: Analysis of relative gene expression data using real-time quantitative PCR and the 2(-Delta Delta $\mathrm{C}(\mathrm{T})$ ) method. Methods 50: 402-408, 2001. 
21. Tekcham DS and Tiwari PK: Non-coding RNAs as emerging molecular targets of gallbladder cancer. Gene 588: 79-85, 2016.

22. Oliveto S, Mancino M, Manfrini N and Biffo S: Role of microRNAs in translation regulation and cancer. World J Biol Chem 8: 45-56, 2017.

23. Yu J, Zhang W, Tang H, Qian H, Yang J, Zhu Z, Ren P and Lu B: Septin 2 accelerates the progression of biliary tract cancer and is negatively regulated by mir-140-5p. Gene 589: 20-26, 2016 .

24. Williams TM, Majithia L, Wang SJ and Thomas CR Jr: Defining the role of adjuvant therapy: Cholangiocarcinoma and gall bladder cancer. Semin Radiat Oncol 24: 94-104, 2014

25. Phelip JM, Vendrely V, Rostain F, Subtil F, Jouve JL, Gasmi M, Michel P, Le Malicot K, Smith D, Seitz JF, et al: Gemcitabine plus cisplatin versus chemoradiotherapy in locally advanced biliary tract cancer: Fédération Francophone de Cancérologie Digestive 9902 phase II randomised study. Eur J Cancer 50 2975-2982, 2014.
26. Valle J, Wasan H, Palmer DH, Cunningham D, Anthoney A, Maraveyas A, Madhusudan S, Iveson T, Hughes S, Pereira SP, et al: Cisplatin plus gemcitabine versus gemcitabine for biliary tract cancer. N Engl J Med 362: 1273-1281, 2010.

27. Li X, Yang L, Shuai T, Piao T and Wang R: miR-433 inhibits retinoblastoma malignancy by suppressing Notch1 and PAX6 expression. Biomed Pharmacother 82: 247-255, 2016.

28. Liang T, Guo Q, Li L, Cheng Y, Ren C and Zhang G: MicroRNA-433 inhibits migration and invasion of ovarian cancer cells via targeting Notch1. Neoplasma 63: 696-704, 2016. 